\title{
Dynamic Characteristics of Electrostatically Actuated Microbeams with Slant Crack
}

\author{
Han Zhou, Wen-Ming Zhang, Zhi-Ke Peng, and Guang Meng \\ State Key Laboratory of Mechanical System and Vibration, School of Mechanical Engineering, Shanghai Jiao Tong University, \\ 800 Dongchuan Road, Shanghai 200240, China
}

Correspondence should be addressed to Wen-Ming Zhang; wenmingz@sjtu.edu.cn

Received 14 September 2014; Accepted 18 December 2014

Academic Editor: Anaxagoras Elenas

Copyright (C) 2015 Han Zhou et al. This is an open access article distributed under the Creative Commons Attribution License, which permits unrestricted use, distribution, and reproduction in any medium, provided the original work is properly cited.

\begin{abstract}
An improved model of the slant crack on a microbeam is presented. Based on fracture mechanics, the rotation coefficient for the slant crack is derived as a massless rotational spring accounting for the additional stress intensity factors generated by the orientation of the crack compared to the transverse crack. Comparisons between microbeams with a slant crack of different geometry parameters (slant angle, depth ratio, and crack position) are investigated with regard to the dynamic mechanical behaviors and nonlinear response. By presenting a mathematical modeling, the effects of the slant crack and the electric actuation of an electrostatically actuated fixed-fixed microbeam on the dynamic characteristics are examined in detail. It is shown that the crack position has more significant influence on the pull-in voltage value than the slant angle or the depth ratio. Approaching the slant crack to the fixed end or enlarging the external incentives amplifies the nonlinearity of the microbeam system, while the effects of depth ratio and slant angle are dependent on the crack position. The resonance frequency and the resonance amplitude are affected as well.
\end{abstract}

\section{Introduction}

With the advantages in miniaturizing reducing cost and energy consumption, the microelectromechanical systems (MEMS) are a growing industry finding their application in different fields such as resonators for sensing $[1,2]$ and electric filtering applications $[3,4]$. Microbeams such as cantilever and doubly clamped beam are the major components of MEMS devices, and the preferred actuation method is always the electric actuation. In an electrostatically actuated microbeam, an air gap capacitor, composed of a movable microbeam (upper electrode) and a fixed (lower) electrode, is connected to a voltage source. This generates electric field and electrostatic force. The elastic force grows linearly with displacement, whereas the electrostatic force grows inversely proportional to the square of the distance when a potential difference is applied between the two electrodes. Consequently, the moveable beam deforms, and the displacement grows when the voltage is increased until at one point the growth rate of the electrostatic force exceeds than the elastic force. The system cannot reach a force balance without a physical contact, and the pull-in instability occurs. This phenomenon is known as "pull-in," and the critical voltage is the pull-in voltage. The reason for this phenomenon is the electrostatic force nonlinearity $[5,6]$.

In order to avoid or use the characteristic of the phenomenon, it is essential to study the pull-in. The pull-in instability is firstly found out in 1967 [7], and later Bernstein et al. [8] proved the existence of a bifurcation point at pullin, where the microbeam deflection becomes unstable, and then Pelesko [9] proved the uniqueness property of the point. A review including the overview of the pull-in phenomenon in electrostatically actuated MEMS and NEMS devices and the physical principals of the pull-in instability provide a comprehensive understanding of the phenomenon [10]. In recent decades, different models have been developed to solve the pull-in problems, such as the nonlinear model of a beam including the electrostatic force, midplane stretching, and applied axial load [11, 12]. In the same time, different analytical numerical methods are improved, such as the shooting method [11], the differential quadrature method (DQM) [13], 
and the combination of step-by-step linearization method (SSLM) and Galerkin-based reduced order model [6].

Another important problem generated during the machining process is the fatigue crack [14]. The existence of the crack was found to induce considerable local flexibility due to the strain energy concentration in the vicinity of the crack tip under load. Moreover, the crack will open or close in time depending on the loading conditions and vibration amplitude, which will change the dynamic characteristics. Eventually, the detection of the structural flaw can be available through measuring such changes. Moreover, a lot of analytical, numerical, and experimental investigations are reported now. Whether the crack is an open or a closed one depends on the combination of the static deflection of the cracked beam caused by some loading components such as the residual loads and the structure weights with the vibration effect. In detail, the crack remains open all the time, or it opens and closes regularly when the static deflection is larger than the vibration amplitudes. But if the static deflection is small, then the crack will open and close in time depending on the vibration amplitude. In these two cases, the former system is linear, while the latter one is nonlinear. So most of the researchers made the assumption that the crack in a structural element is open and remains open during vibration in their work, in order to avoid the complexities that resulted from the nonlinear characteristics presented by introducing a breathing crack (a crack which opens and closes during vibration) [15-17].

Many researchers are focused on developing suitable models to describe the effect of damage on the beam-like structures, and different approaches for crack modeling have been reported. The approaches can be generally divided into three categories [18]: spring models or elastic hinges [19], local stiffness reduction [20], and finite element models [21]. According to Friswell and Penny in [22], simple models of crack flexibility based on beam elements are adequate compared with other approaches, and those models belong to the category relying on the spring models. In particular, the model of crack as an internal hinge endowed with a rotational spring connecting the two adjacent beam segments is of high accuracy.

Based on that, the crack is modeled as an equivalent massless rotational spring with a local compliance, and this method was often used to quantify the relation between the applied load and the strain concentration around the tip of the crack in a macroscopic way $[23,24]$. With improvement of the method in recent years, the crack is substituted by the additional local compliance of a cracked beam with relation to the strain energy concentration as well as to the stress intensity factor, and a lot of results both analytical and experimental are gained under different loading and geometry for a number of cases. Most researchers assumed the crack to be open but not close, which guaranteed a constant stiffness and frequency shift during vibration. Lin et al. [25] investigated the cracked beams model as a function of the crack depth as well as the consequently dynamic behaviors and the stability characteristics. Furthermore, Rubio and Fernández-Sáez studied the applicability of different approximate closed-form solutions to evaluate the natural frequencies for bending vibrations of simply supported Euler-Bernoulli cracked beams [26]. Motallebi et al. [6] presented investigation of the effects of the geometry parameters, such as the crack depth, crack position, and the crack number, of open crack on the static and dynamic pull-in voltages of the microbeams under different support methods. Afshari and Inman presented results that the proposed crack modeling approach as a massless rotational spring was beneficial as it provided twice-differentiable mode shapes for the cracked beam and can be used as trial functions in the assumed mode approximations [27].

Since resonant sensors are important components in micromechanical devices, the investigations into the frequency response of a resonant microbeam to an electric actuation are of big significance [28]. As reported, frequency of a resonant microbeam is very sensitive to the axial strain induced by external loads, such as pressure, temperature, force, and acceleration, and consequently causes a shift in its natural frequencies. Such shift is generally converted to a digital signal related to the physical quantity being measured [29]. Additionally, some reports show that some other factors such as squeeze-film damping [30] and the elasticity of the microbeam supports can also originate the frequency shift [31]. Alsaleem et al. [32] presented results of modeling, analysis and experimental investigation for nonlinear resonances, and the dynamic pull-in instability in electrostatically actuated resonators. Caddemi et al. [18] studied the nonlinear dynamic response of the Euler-Bernoulli beam in presence of multiple concentrated switching cracks (i.e., cracks that are either fully open or fully closed). Besides the transverse crack analyses, the dynamic characteristics of slant crack attract attention of researchers. But most such study is based on the vibrations analysis of rotors with slant crack, for example, $[33,34]$. Little investigation of effect of slant crack on the dynamic response of microbeams is carried out.

In this paper, an improved model of beam with slant open crack is presented as a massless rotational spring. It is based on a continuous beam model and the stress and strain energy analysis of the vicinity of the crack. The combinatorial method of SSLM and Galerkin-based reduced order model is used to investigate the static response of MEMS devices, and the fixed-fixed beam model is applied. The natural frequencies and corresponding cracked mode shapes of the microbeam are calculated with the usage of the transfer matrix method under the boundary and patching conditions. And then the comparisons of the dynamic vibration behaviors and the pull-in voltages of beams with different geometry parameters of slant crack are shown. The effects of the crack depth, crack slant angle, and crack position on the dynamic vibration behaviors and the pull-in voltages are discussed. Finally, an investigation into the dynamic response of the microbeam is presented by using the multiple scales method, and the effects of the crack parameters and the electric actuation are discussed on the vibration nonlinearity.

\section{Mathematical Modeling}

Figure 1 shows the schematic of an electrostatically actuated fixed-fixed microbeam with a slant crack on the surface. 


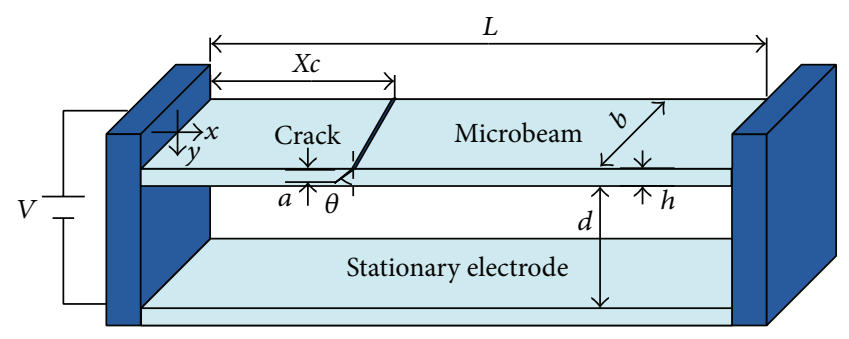

(a)

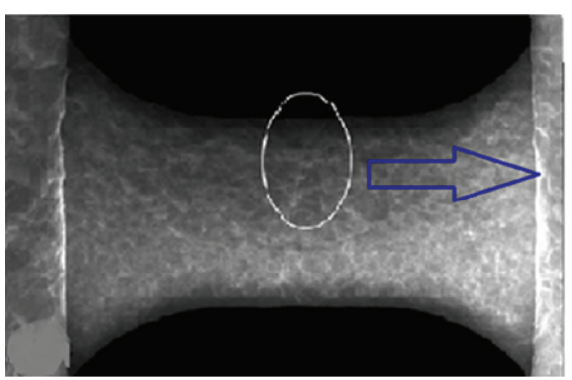

(c)

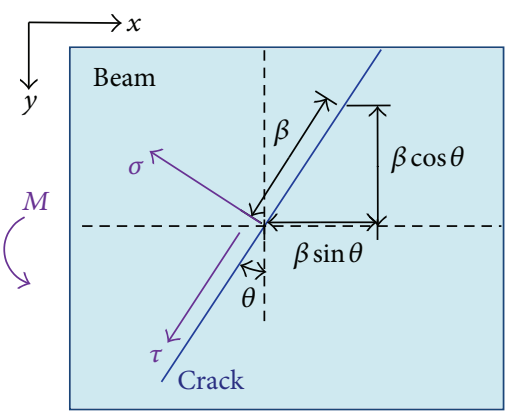

(b)

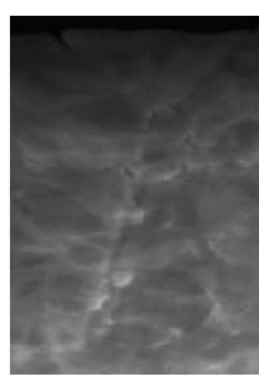

FIGURE 1: Schematic of a microbeam with a slant crack on the surface. (a) The mathematical modeling of an electrostatically actuated fixed-
fixed microbeam with a slant crack. (b) Top view of stress components analysis of the microbeam loaded with a bending moment $M$. (c) Optical image of an actual beam specimen with a slant crack located at the center of a MEMS fatigue device and anchored to two lateral plates acting as electrostatic actuators [35].

The specimen is the small suspended fixed-fixed microbeam with a rectangular cross section located at the center of a MEMS device, which is fabricated using the gold electrodeposition technique [35]. The beam specimen is anchored to two lateral plates acting as electrostatic actuators (Figure 1(c)). When the plates are actuated, the beam structure deforms and undergoes a tensile load. The magnified section reveals the formation of intruded and extruded sections and the propagated crack. Figure 1(a) depicts the simplified mathematical modeling of the electrostatically actuated fixed-fixed microbeam, including a suspended elastic beam with an applied electrostatic force and another beam fixed at both ends to the ground conductor. The upper beam is suspended over a dielectric film deposited on top of the center conductor, which will be pulled down for the electrostatic force when a voltage is applied between the upper and lower electrodes. The microbeams are considered as Euler-Bernoulli beams of length $L$, width $b$, thickness $h$, density $\rho$, Young's modulus $\widetilde{E}$, Poisson's ratio $\nu$, and $\bar{E}=$ $\widetilde{E} /\left(1-v^{2}\right)$. On the surface of the beam, an slant crack was located at point $X c$, having a depth $a$ oriented at an angle of $\theta$ relative to the normal direction of the beam, the depth ratio of $\gamma=a / h$. $d$ and $\varepsilon$ are initial gap and dielectric constant, respectively. $\widetilde{x}$ and $\widetilde{w}(\widetilde{x}, \widetilde{t})$ denote the coordinate along the length of the microbeam with its origin at the left end and the transverse displacement of the beam, respectively. Now, the entire beam is divided into two parts with length of $X c$ and $L-X c$, respectively [6], and the global nonlinear system can be separated into two linear subsystems joined by an additional local stiffness discontinuity. According to the theory of fracture mechanics, the additional rotation coefficient can be derived from the strain energy release rate $J$ and the strain energy $U$. For plane strain, the expression of $J, U$ and the relation of them are $[36,37]$

$$
\begin{gathered}
J=\frac{1}{\bar{E}}\left(K_{I}^{2}+K_{I I}^{2}+(1+v) K_{I I}^{2}\right) \\
U=\iint_{A} J d A \\
\Theta=\frac{\partial U^{2}}{\partial P^{2}},
\end{gathered}
$$

where $A$ is the area of the crack. $K_{I}, K_{I I}, K_{I I I}$ represent the stress intensity factors (SIFs) corresponding to opening, sliding, and tearing mode of crack displacement, respectively. $\Theta$ is the additional rotation in the slope due to the existence of the crack, and $P$ is the force loaded on the beam.

The SIFs are derived as follows. To simplify the problem, the slant-cracked beam is loaded with pure bending moment $M$. As shown in Figure 1(b), compared to a transverse crack, the bending $M$ leads to more numbers of stress components responsible for the opening and tearing mode of crack displacement. The stress components include the shear stress $\tau$ and the normal stress $\sigma$, which cause tearing mode and opening mode of the crack, respectively. According to stress analysis [38], it can be gotten that

$$
\begin{gathered}
\sigma_{0}=\frac{M}{I} \beta ; \quad \tau_{0}=0 ; \quad \sigma_{c}=\sigma_{0} \cos ^{2} \theta ; \\
\tau_{c}=\sigma_{0} \cos \theta \sin \theta,
\end{gathered}
$$


where $\beta$ is the distance from the centre point of the crack along the crack edge. $\sigma_{0}, \tau_{0}$ and $\sigma_{c}, \tau_{c}$ are the stress components in the transverse crack surface and the slant crack surface, respectively. And $I=1 / 12 b h^{3}$ is the inertia moment of microbeam cross section. To simplify the calculation, we take the largest stress, which means $\sigma_{0}=6 \mathrm{M} / \mathrm{bh}^{2}$.

Then, SIFs for opening, sliding, and tearing mode can be expressed as

$$
\begin{gathered}
K_{I}^{0}=\sigma_{0} \sqrt{\pi \alpha} F_{2}, \quad K_{I I}^{0}=K_{I I I}^{0}=0 ; \\
K_{I}^{c}=\sigma_{c} \sqrt{\pi \alpha} F_{2}, \quad K_{I I}^{c}=0, \quad K_{I I I}^{c}=\tau_{c} \sqrt{\pi \alpha} F_{I I I},
\end{gathered}
$$

where $K_{I}^{0}, K_{I I}^{0}$, and $K_{I I I}^{0}$ represent SIFs related to the transverse crack, while $K_{I}^{c}, K_{I I}^{c}$, and $K_{I I I}^{c}$ are related to the slant crack, and one has

$$
\begin{gathered}
F_{2}\left(\frac{a}{h}\right) \\
=\sqrt{\frac{2 h}{\pi a} \tan \left(\frac{\pi a}{2 h}\right)}\left(\frac{0.923+0.199[1-\sin (\pi a / 2 h)]^{4}}{\cos (\pi a / 2 h)}\right) \\
F_{I I I}\left(\frac{a}{h}\right)=\sqrt{\frac{2 h}{\pi a} \tan \left(\frac{\pi a}{2 h}\right) .}
\end{gathered}
$$

With the expressions of SIFs derived above, synthesizing (1) to (3), adopting the correction function given by Anifantis and Dimarogonas and Taylor's series expansion [23], the nondimensional rotation expression is obtained as

$$
\begin{gathered}
\Theta=6 \pi\left(1-v^{2}\right)\left(\frac{h}{L}\right)\left[\cos ^{3}(\theta) f_{1}(\gamma)\right. \\
\left.+(1+v) \cos (\theta) \sin ^{2}(\theta) f_{2}(\gamma)\right] \\
f_{1}(\gamma)=0.6348 \gamma^{2}-1.035 \gamma^{3}+3.7201 \gamma^{4}-5.1773 \gamma^{5} \\
+7.553 \gamma^{6}-7.332 \gamma^{7}+2.4909 \gamma^{8} \\
f_{2}(\gamma)=0.2026 \gamma^{2}+0.03378 \gamma^{4}+0.009006 \gamma^{6}+0.002734 \gamma^{8} .
\end{gathered}
$$

The microbeam is subject to a viscous damping due to squeeze-film damping. And this effect is approximated by an equivalent damping coefficient $\widetilde{c}$ per unit length $[39,40] . \widetilde{N}_{a}$ and $\widetilde{N}_{r}$ are the stretching and residual forces, respectively. Thus, the nondimensional governing equation of the transverse vibration of the beam can be given by [6]

$$
\frac{\partial^{4} w}{\partial x^{4}}+\frac{\partial^{2} w}{\partial t^{2}}+c \frac{\partial w}{\partial t}-\left(N_{a}+N_{r}\right) \frac{\partial^{2} w}{\partial x^{2}}=\alpha\left[\frac{V(t)}{(1-w)}\right]^{2} .
$$

The boundary conditions of the fixed-fixed microbeam are as follows:

$$
w(0, t)=w(1, t)=\frac{\partial w}{\partial x}(0, t)=\frac{\partial w}{\partial x}(1, t)=0,
$$

where the nondimensional variables and parameters are

$$
\begin{gathered}
w=\frac{\widetilde{w}}{d}, \quad x=\frac{\widetilde{x}}{L}, \quad t=\frac{\widetilde{t}}{t^{*}}, \\
\alpha=\frac{6 \varepsilon L^{4}}{\bar{E} h^{3} d^{3}}, \quad t^{*}=\sqrt{\frac{\rho b h L^{4}}{\bar{E} I}} \\
N_{r}=\frac{12 \widetilde{N}_{r} L^{2}}{\bar{E} h^{3} b}, \quad c=\frac{12 \widetilde{c} L^{4}}{\bar{E} h^{3} b t^{*}}, \\
N_{a}=6\left(\frac{d}{h}\right)^{2} \int_{0}^{1}\left(\frac{\partial w}{\partial x}\right)^{2} d x .
\end{gathered}
$$

By dropping the time dependence of (6), the governing equation can be rewritten as

$$
\frac{\partial^{4} w}{\partial x^{4}}-\left(N_{a}+N_{r}\right) \frac{\partial^{2} w}{\partial x^{2}}=\alpha\left[\frac{V}{(1-w)}\right]^{2} .
$$

\section{Numerical Analysis}

In order to study the effect of the slant crack on the local stiffness discontinuity, each segment divided by the crack can be considered as a separate beam under undamped free vibration, with nondimensional equation form as follows:

$$
\frac{\partial^{4} w_{i}}{\partial x^{4}}+\frac{\partial^{2} w_{i}}{\partial t^{2}}=0, \quad i=1,2 .
$$

Using the separable solutions, $w_{i}(x, t)=Y_{i}(x) e^{j \omega t}$, in (10) leads to the associated eigenvalue problem:

$$
\frac{\partial^{4} Y_{i}}{\partial x^{4}}-\Lambda^{4} Y_{i}=0, \quad i=1,2
$$

with patching conditions as [25]

$$
\begin{gathered}
Y_{1}\left(l c^{-}\right)=Y_{2}\left(l c^{+}\right), \quad Y_{1}^{\prime \prime}\left(l c^{-}\right)=Y_{2}^{\prime \prime}\left(l c^{+}\right) \\
Y_{1}^{\prime \prime \prime}\left(l c^{-}\right)=Y_{2}^{\prime \prime \prime}\left(l c^{+}\right), \quad Y_{2}^{\prime}\left(l c^{+}\right)-Y_{1}^{\prime}\left(l c^{-}\right)=\Theta Y_{2}^{\prime \prime}\left(l c^{+}\right),
\end{gathered}
$$

where $\Lambda^{4}=\omega^{2}$ and $l c=X c / L$, with $\omega$ being the nondimensional nature frequency and $\Lambda$ being the frequency parameter.

The general solution of the eigenvalue problem in (11) with boundary conditions in (7) and patching conditions in (12) is written as

$$
\begin{aligned}
Y_{1}(x)= & A_{1} \sin [\Lambda x]+B_{1} \cos [\Lambda x]+C_{1} \sinh [\Lambda x] \\
& +D_{1} \cosh [\Lambda x], \quad(0 \leq x \leq l c) \\
Y_{2}(x)= & A_{2} \sin [\Lambda(x-l c)]+B_{2} \cos [\Lambda(x-l c)] \\
& +C_{2} \sinh [\Lambda(x-l c)]+D_{2} \cosh [\Lambda(x-l c)], \\
& (l c \leq x \leq 1),
\end{aligned}
$$

where $A_{i}, B_{i}, C_{i}, D_{i}(i=1,2)$ are constants associated with the $i$ th segment. And along with the process, the square of 
the series value of $\Lambda$ obtained corresponds to the nondimensional natural frequencies of associated modes.

Because of the nonlinearity of the static equation, the numerical solution is complicated and time consuming, and the direct application of the Galerkin method or finite difference method creates a set of nonlinear algebraic equations. In this paper, a method of two steps is used. In the first step, the SSLM is used $[8,41]$, and in the second step, the Galerkin method for solving the linear equation obtained is applied. Using the SSLM, it is assumed that $w^{k}$ is the displacement of beam due to the applied voltage $V^{k}$. Therefore, by increasing the applied voltage to a new value, the displacement can be written as [6]

$$
w_{s}^{k+1}=w_{s}^{k}+\delta w=w_{s}^{k}+\psi(x)
$$

when

$$
V^{k+1}=V^{k}+\delta V
$$

So, the equation of the static deflection of the fixed-fixed microbeam (equation (9)) can be rewritten in the step of $k+1$ as follows:

$$
\frac{\partial^{4} w_{s}^{k+1}}{\partial x^{4}}-\left(N_{a}^{k+1}+N_{r}\right) \frac{\partial^{2} w_{s}^{k+1}}{\partial x^{2}}=\alpha\left[\frac{V}{\left(1-w_{s}^{k+1}\right)}\right]^{2} .
$$

By considering the small value of $\delta V$, it is expected that $\psi$ would be small enough. Thus by using the calculus of variation theory and Taylor's series expansion about $w^{k}$ in (16) and applying the truncation to its first order for suitable value of $\delta V$, it is possible to obtain the desired accuracy. The linearized equation for calculating $\psi$ can be expressed as

$$
\begin{gathered}
\frac{d^{4} \psi}{d x^{4}}-\left(N_{a}^{k}+\delta N_{a}+N_{r}\right) \frac{d^{2} \psi}{d x^{2}}-\left.\delta N_{a} \frac{d^{2} w}{d^{2} x}\right|_{\left(w^{k}, V^{k}\right)} \\
-2 \frac{\alpha\left(V^{k}\right)^{2}}{\left(1-w_{s}^{k}\right)^{3}} \psi-2 \frac{\alpha V^{k} \delta V}{\left(1-w_{s}^{k}\right)^{2}}=0,
\end{gathered}
$$

where the variation of the hardening term based on the calculus of variation theory can be expressed as $\delta N_{a}=$ $\int_{0}^{1} \psi(x) d^{2} w /\left.d^{2} x\right|_{\left(w^{k}, V^{k}\right)} d x$. Similarly, because of the small value of $\delta V$ and $\psi$, the value of multiplying $\delta N_{a}$ by $d^{2} \psi / d x^{2}$ would be small enough that can be neglected.

The linear differential equation obtained is solved by the Galerkin method, and $\psi(x)$ based on function spaces can be expressed as

$$
\psi(x)=\sum_{j=1}^{n} a_{j} Y_{j}(x)
$$

where $Y_{j}(x)$ is selected as the $j$ th undamped linear mode shape of the cracked microbeam. $\psi(x)$ is approximated by truncating the summation series to a finite number $n$ in this work.

Substituting (18) into (17) and multiplying by $Y_{i}(x)$ as a weight function in the Galerkin method and then integrating the outcome from $x=0$ to 1 , a set of linear algebraic equations can be obtained as follows:

$$
\sum_{j=1}^{n} K_{i j} a_{j}=F_{i} \quad(i=1,2, \ldots, n),
$$

where

$$
\begin{gathered}
K_{i j}=K_{i j}^{m}+K_{i j}^{a}-K_{i j}^{e}, \quad K_{i j}^{m}=\int_{0}^{1} Y_{i} Y_{j}^{(4)} d x \\
K_{i j}^{a}=\int_{0}^{1} Y_{i}\left[\left(N_{a}^{k}+N_{r}\right) Y_{j}^{\prime \prime}\right. \\
\left.+\left.\left(\left.\int_{0}^{1} Y_{i} \frac{d^{2} \omega}{d x^{2}}\right|_{\omega^{k}, V^{k}} d x\right) \frac{d^{2} \omega}{d x^{2}}\right|_{\omega^{k}, V^{k}}\right] d x \\
K_{i j}^{e}=2 \alpha\left(V^{k}\right)^{2} \int_{0}^{1} \frac{Y_{i} Y_{j}}{\left(1-\omega^{k}\right)^{3}} d x \\
F_{i}=2 \alpha\left(V^{k+1}-V^{k}\right) \int_{0}^{1} \frac{Y_{i}(x)}{\left(1-\omega^{k}\right)^{2}} d x .
\end{gathered}
$$

Considering the resonant microbeam (see Figure 1) actuated by an electric load that is composed of a DC component (polarization voltage) $V_{p}$ and an AC component $v_{\mathrm{ac}}$, the voltage signal can be given by

$$
V(t)=V_{p}+v_{\mathrm{ac}} \cos \left(\omega_{e} t\right),
$$

where $\omega_{e}$ is the excitation frequency and $V_{p} \gg v_{\mathrm{ac}}$.

In order to investigate the dynamic behavior of the microbeams more simply, the linearization of the right part of the governing equation (6) can be written as

$$
\begin{aligned}
{\left[\frac{V(t)}{(1-w)}\right]^{2}=} & {\left[V_{p}^{2}+2 V_{p} v_{\mathrm{ac}} \cos \left(\omega_{e} t\right)+v_{\mathrm{ac}}^{2} \cos ^{2}\left(\omega_{e} t\right)\right] } \\
& \cdot\left(1+2 w+3 w^{2} \cdots\right) \\
= & V_{p}^{2}\left(1+2 w+3 w^{2}\right)+2 V_{p} v_{\mathrm{ac}} \cos \left(\omega_{e} t\right),
\end{aligned}
$$

where the term involving $v_{\text {ac }}^{2}$ is dropped because typically $v_{\mathrm{ac}} \ll V_{p}$ in resonant sensors. Using the Rayleigh-Ritz method, assuming the bending deflection of the beam to be of the form $w(x, t)=Y(x) * u(t)$ as analyzed previously, and substituting (22) into (6), we can get the governing equation as a single degree-of-freedom Duffing equation as follows:

$$
\ddot{u}+\omega_{0}^{2} u-\hbar u^{3}=\lambda \dot{u}+\ell u^{2}+\chi \cos \left(\omega_{e} t\right)+\kappa,
$$

where $u(t), \dot{u}(t), \ddot{u}(t)$ are the displacement, speed, and acceleration of the center layer of the beam. And one has

$$
\begin{gathered}
\omega_{0}^{2}=a_{1}-N_{r} a_{2}-2 \alpha V_{p}^{2}, \quad \hbar=\frac{6 d^{2} a_{3}}{h^{2}} \\
\lambda=-c, \quad \ell=3 \alpha b_{3} V_{p}^{2}, \quad \chi=2 \alpha b_{1} v_{\mathrm{ac}} V_{p}^{2}, \\
\kappa=\alpha b_{1} V_{p}^{2},
\end{gathered}
$$


where

$$
\begin{gathered}
a_{1}=\int_{0}^{1} Y^{(4)}(x) Y(x) d x, \quad a_{2}=\int_{0}^{1} Y^{(2)}(x) Y(x) d x \\
a_{3}=a_{2} \int_{0}^{1}\left[Y^{\prime}(x)\right]^{2} d x, \quad b_{i}=\int_{0}^{1} Y^{i}(x) d x, \\
(i=1,2,3), \quad b_{2}=1 .
\end{gathered}
$$

To calculate the nonlinear response of the electrostatically actuated microbeam given by (23), the multiple scale perturbation theory is employed. The response to the primary resonance excitation of its first mode is analyzed because it is the case that is used in resonator applications. Still, the analysis is general and can be used to study nonlinear responses of the beam to a primary resonance of any of its modes. The excitation frequency $\omega_{e}$ is usually tuned close to the fundamental nature frequency of mechanical vibrations, namely, $\omega_{e}=\omega_{0}+\eta \sigma$, where $\eta$ is a dimensionless small parameter and $\sigma$ is a small detuning parameter. By redefining the parameters as $\hbar=\eta \hbar, \lambda=\eta \lambda, \ell=\eta \ell, \chi=\eta \chi$, and $\kappa=\eta \kappa$, (23) can be written as

$$
\ddot{u}+\omega_{0}^{2} u=\eta\left[\hbar u^{3}+\lambda \dot{u}+\ell u^{2}+\chi \cos \left(\omega_{0}+\varepsilon \sigma\right) t+\kappa\right] .
$$

To solve (26), two time scales, $T_{0}=t$ and $T_{1}=\eta t$, are introduced, and the first-order uniform solution is given in the form

$$
u(t, \eta)=u_{0}\left(T_{0}, T_{1}\right)+\eta u_{1}\left(T_{0}, T_{1}\right) .
$$

Substituting (27) into (26) and equating coefficients of like powers of $\varepsilon$, the linear partial differential equations of order $\eta^{0}$ and order $\eta^{1}$ are obtained as

$$
\begin{gathered}
D_{0}^{2} u_{0}+\omega_{0}^{2} u_{0}=0 \\
D_{0}^{2} u_{1}+\omega_{0}^{2} u_{1}=-2 D_{0} D_{1} u_{0}+\hbar u_{0}^{3}+\lambda D_{0} u_{0}+\ell u_{0}^{2} \\
+\chi \cos \left(\omega_{0}+\eta \sigma\right) T_{0}+\kappa,
\end{gathered}
$$

where $D_{0}=\partial / \partial T_{0}$ and $D_{1}=\partial / \partial T_{1}$. The general solution of the first equation of (28) can be written as

$$
u_{0}\left(T_{0}, T_{1}\right)=a\left(T_{0}\right) \cos \left[\omega_{0} T_{0}+\delta\left(T_{1}\right)\right]=A\left(T_{1}\right) e^{j \omega_{0} T_{0}}+c c,
$$

where $A\left(T_{1}\right)=a\left(T_{0}\right) e^{j \delta\left(T_{1}\right)} / 2$ and cc denotes a complex conjugate. Equation (29) is then substituted into the second equation of (28), and the trigonometric functions are expanded. The elimination of the secular terms yields two first-order nonlinear ordinary differential equations that allow for a stability analysis. Furthermore, the modulation of the response with the amplitude $a$ and phase $\delta$ is given by

$$
\begin{gathered}
D_{1} a=\frac{\lambda a}{2}+\frac{\chi}{2 \omega_{0}} \sin \varphi \\
a D_{1} \delta=-\left(\frac{3 \hbar a^{3}}{8 \omega_{0}}+\frac{\chi}{2 \omega_{0}} \cos \varphi\right),
\end{gathered}
$$

where $\varphi=\sigma T_{1}-\delta$. The steady-state motion occurs when $D_{1} a=D_{1} \varphi=0$, which corresponds to singular points of (30). Then the steady-state frequency response is obtained as

$$
\left[\left(\omega_{e}-\omega_{0}+\frac{3 \hbar A_{0}^{2}}{8 \omega_{0}}\right)^{2}+\left(\frac{\lambda}{2}\right)^{2}\right] A_{0}^{2}=\left(\frac{\chi}{2 \omega_{0}}\right)^{2},
$$

where $A_{0}$ is the resonance amplitude when it is steady-state. While one has $A_{0} \leq \chi /\left(\lambda \omega_{0}\right)$, a function of the independent incentive frequency is found to be

$$
\omega_{e}=\omega_{0}-\frac{3 \hbar}{8 \omega_{0}} A_{0}^{2} \pm \sqrt{\frac{\chi^{2}}{4 \omega_{0}^{2} A_{0}^{2}}-\frac{1}{4} \lambda^{2}} .
$$

Defining nondimensional resonance frequency, $\Omega=\omega_{e} / \omega_{0}$, (32) can be expressed as

$$
\Omega=1-\frac{3 \hbar}{8 \omega_{0}^{2}} A_{0}^{2} \pm \sqrt{\frac{\chi^{2}}{4 \omega_{0}^{4} A_{0}^{2}}-\frac{\hbar^{2}}{4 \omega_{0}^{2}}} .
$$

As indicated in (33), the nondimensional design parameters affect the resonance frequency through changing the effective nonlinearity of the undamped linear mode shape.

The maximum resonance amplitude is reached when the magnitude under the square root is zero [42]. Hence,

$$
A_{\max }=\frac{\chi}{\lambda \omega_{0}}
$$

and the corresponding frequency is

$$
\Omega=1-\frac{3 \hbar}{8 \omega_{0}^{2}} A_{\max }^{2}
$$

The curve determined by (35) is defined as the skeleton line, which manifests the relation between the peak amplitude and the resonance frequency, dominating the shape of the amplitude-frequency response (AFR). The AFR curves indicate the nonlinear characteristics of the microbeam. According to (33) and (35), the AFR curves and the skeleton lines not only are affected by parameters of the beam structure and the slant crack, but also are related to the external incentives, namely, the $\mathrm{DC}$ and $\mathrm{AC}$ voltages.

\section{Results and Discussion}

4.1. Frequency and Mode Shapes. The geometric and material properties of the microbeam are as follows: $L=250 \mu \mathrm{m}$, $b=50 \mu \mathrm{m}, \varepsilon=8.85 \mathrm{PF} / \mathrm{m}, h=3 \mu \mathrm{m}, d=1 \mu \mathrm{m}, E=$ $1.69 \mathrm{GPa}, \rho=2331 \mathrm{~kg} / \mathrm{m}^{3}$, and $\nu=0.06$ [6]. Figure 2 shows the effects of the crack depth ratio for selected crack positions $(l c=0.05,0.25,0.5)$ and slant angles $\left(\theta=0^{\circ}, 20^{\circ}\right)$ on the value of the first four $(n=1-4)$ nature frequencies along the depth ratio. It can be found that increasing the depth ratio will gradually decrease the natural frequencies, with the tendency serving as the confirmation of the EBB theory according to Hasheminejad et al. [43]. Some important observations are found. Increasing the crack depth ratio does not have any impact on the cases of $n=2(l c=0.5)$ and $n=4(l c=0.5)$, 


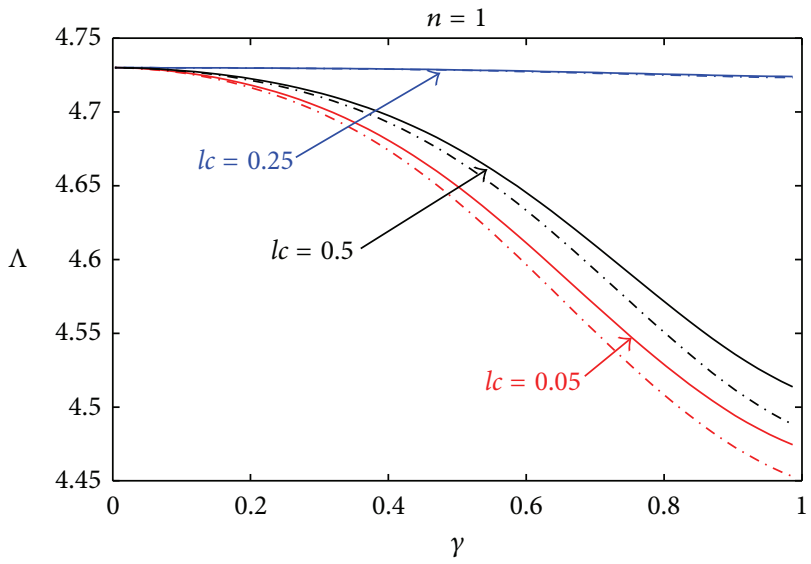

(a)

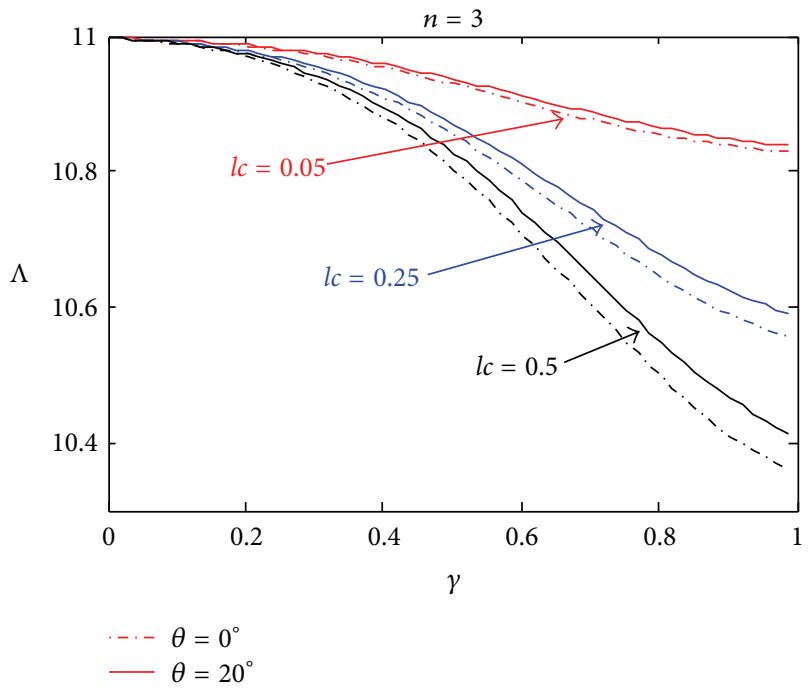

(c)

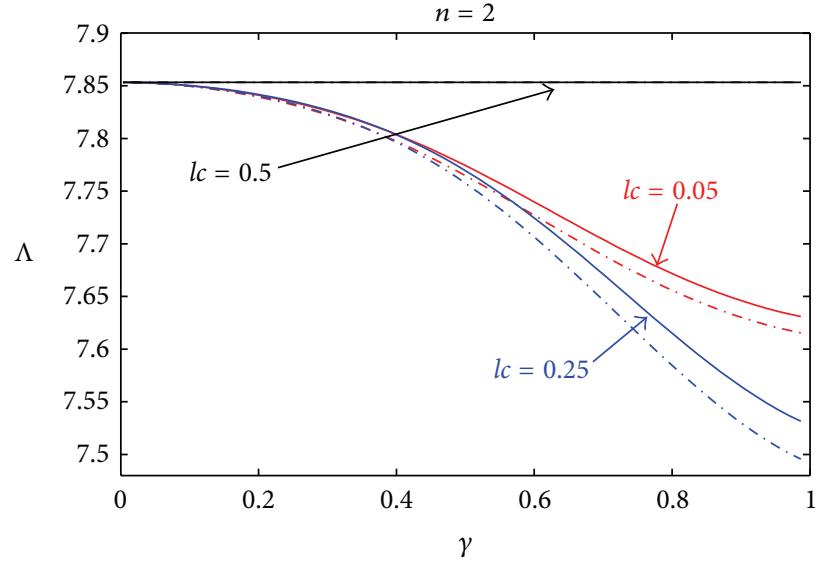

(b)

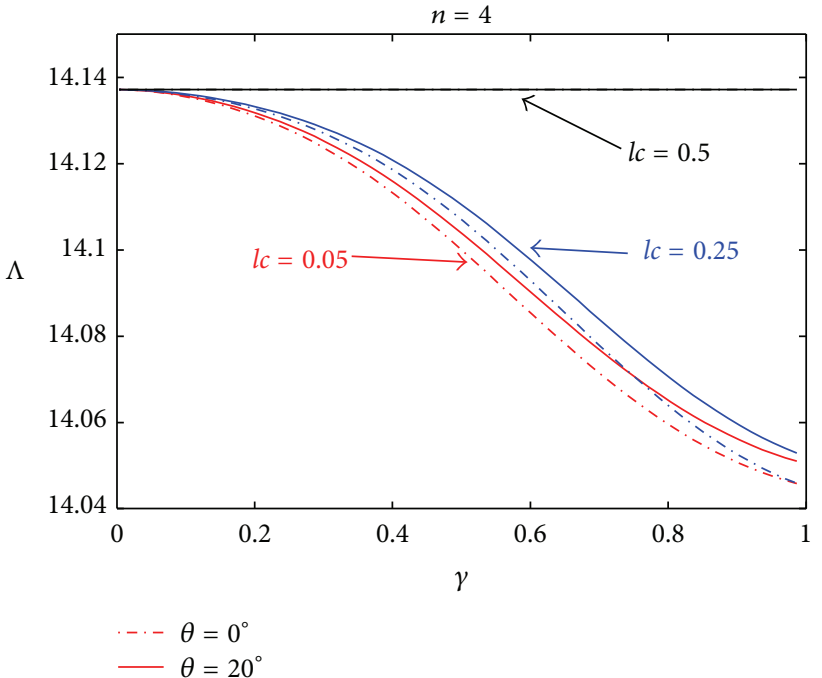

(d)

FIGURE 2: The first four nondimensional natural frequencies of the microbeam along with the depth ratio of the crack for three crack positions and two slant angles.

TABLE 1: Values of the first four frequency parameters for a fixed-fixed beam with different crack positions and crack slant angles. Crack depth ratio $\gamma=0.5$.

\begin{tabular}{|c|c|c|c|c|c|c|c|c|}
\hline \multirow{2}{*}{$\Lambda$} & \multicolumn{4}{|c|}{$l c=0.05$} & \multicolumn{4}{|c|}{$l c=0.5$} \\
\hline & $\theta=0^{\circ}$ & $\theta=15^{\circ}$ & $\theta=30^{\circ}$ & $\theta=45^{\circ}$ & $\theta=0^{\circ}$ & $\theta=45^{\circ}$ & $\theta=30^{\circ}$ & $\theta=45^{\circ}$ \\
\hline$n=1$ & 4.6392 & 4.6452 & 4.6616 & 4.6836 & 4.6673 & 4.6719 & 4.6840 & 4.6995 \\
\hline$n=2$ & 7.7647 & 7.7702 & 7.7855 & 7.8065 & 7.8532 & 7.8532 & 7.8532 & 7.8532 \\
\hline$n=3$ & 10.929 & 10.933 & 10.944 & 10.960 & 10.806 & 10.819 & 10.854 & 10.901 \\
\hline$n=4$ & 14.100 & 14.102 & 14.108 & 14.117 & 14.137 & 14.137 & 14.137 & 14.137 \\
\hline
\end{tabular}

which exhibits the same trend with results presented by Hasheminejad et al. [43]. This can be explained by the fact that none of the crack positions $(l c=0.5)$ lie on the vibration nodes for the beam vibrating in first and third modes $(n=1$, $3)$, while in the case of the second and fourth modes $(n=2$, $4)$, the crack position $(l c=0.5)$ is exactly set on the vibration nodes.

Take the slant angle of the crack into account, the increment in angle leads to a gradual increase of the four nature frequencies, except the special cases mentioned above. Besides, the effect of the crack position on frequency differs in different modes.

More detailed changes of the first four frequency parameters for the fixed-fixed microbeam with different crack slant angles $\theta$ for a crack located at positions $l c=0.05$ and 0.5 are given in Table 1 , and the crack depth ratio $\gamma=0.5$.

The first-mode shapes of the cracked microbeam of different geometry characteristics are displayed in Figure 3. 


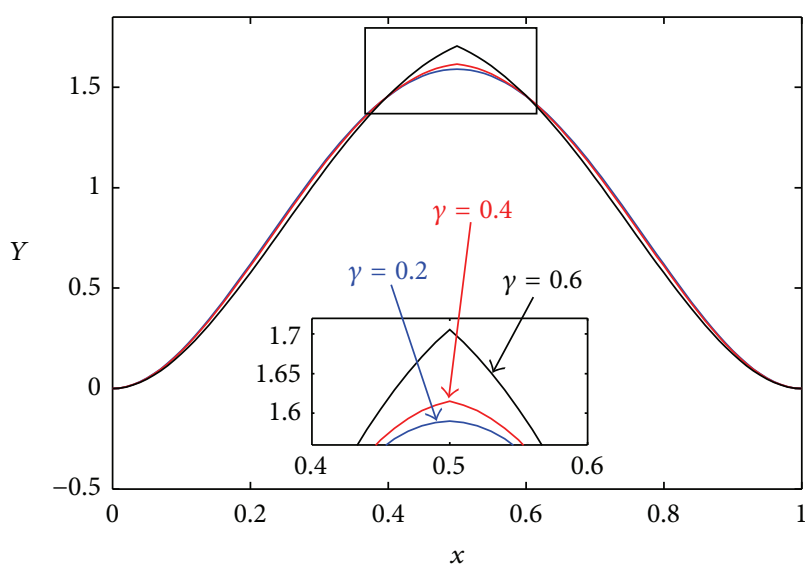

(a)

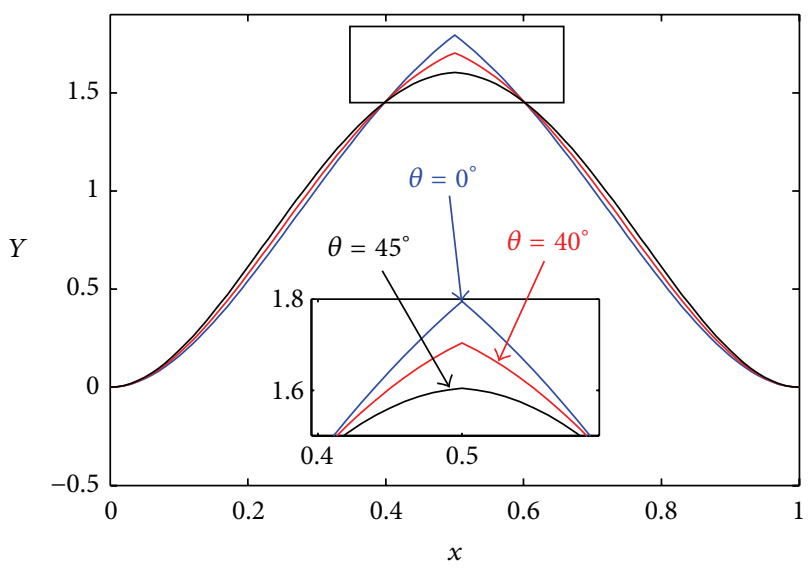

(c)

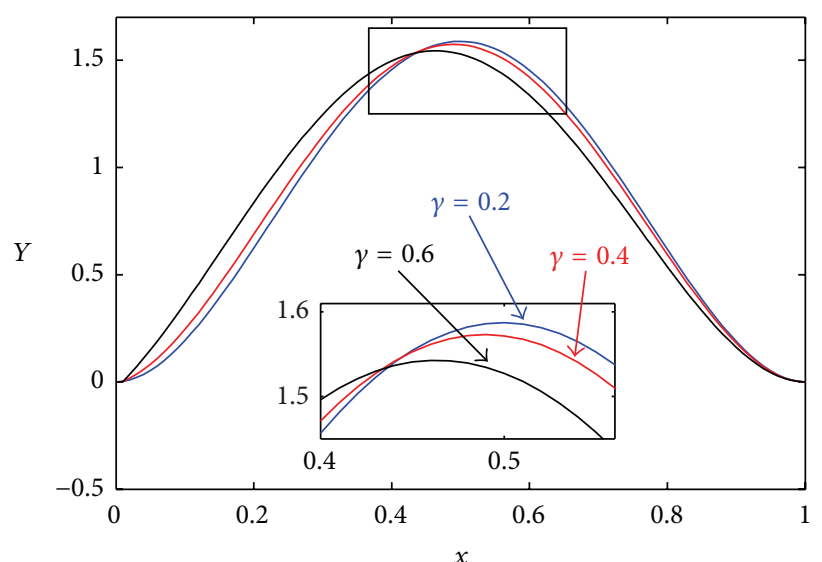

(b)

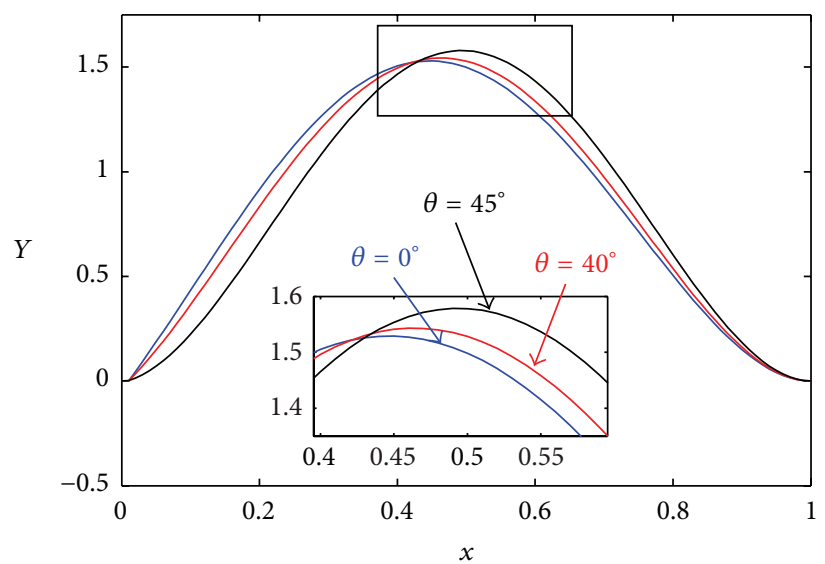

(d)

Figure 3: The effects of the depth ratio $(a, b)$ and the slant angle $(c, d)$ of the slant crack on the first-mode shapes of the beam at different positions. (a) and (c) $l c=0.5$; (b) and (d) $l c=0.05$.

The abscissa is the nondimensional distance of the crack from the left end of the microbeam, and the ordinate is the vibration amplitude of first-mode shape. It manifests that the effects of the slant angle and depth ratio on the firstmode shapes depend on the crack position. For example, the growth in the depth ratio will increase the maximum vibration amplitude along the beam length when the crack is near the center point of the beam $(l c=0.5$, see Figure $3(\mathrm{a}))$, but it will lead the inverse trend when the crack is located at the end part $(l c=0.05$, see Figure $3(\mathrm{~b}))$. Similarly, when it comes to the slant angle (see Figures 3(c) and 3(d)), the angle increment causes decrease in the maximum vibration amplitude when the crack is located near the center part of the beam, but it increases when it is near the end. The effect differences in vibration amplitude are in good agreement with the ones in the nature frequency. Additionally, the maximum vibration amplitude is larger when the crack position gets near the center part.

4.2. Pull-In Voltage Analysis. In the pull-in voltage analysis, voltages will differ when different voltage increment is applied, but the difference is small enough to be neglected.

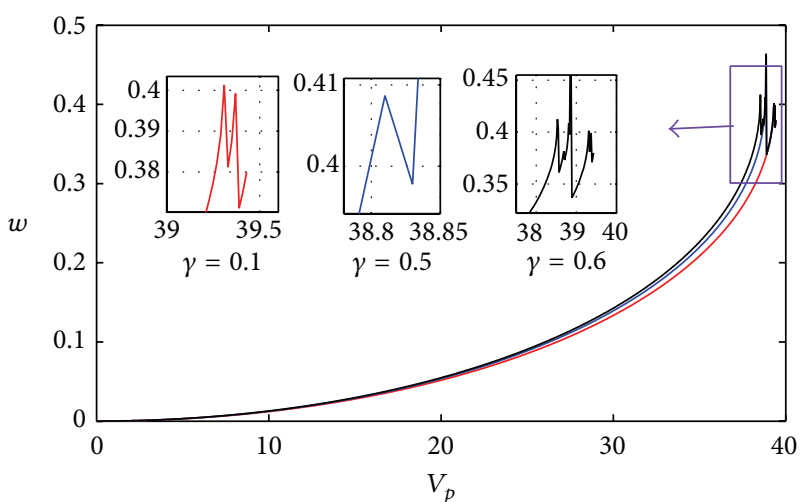

FIGURE 4: The displacement of center point of the beam with the DC voltage for case of position 0.5 , slant angle $45^{\circ}$, and a static voltage increment of $0.02 \mathrm{~V}$, for three depth ratios $(0.1,0.5$, and 0.6$)$.

Just as seen in Figure 4, the abscissa is the value of the applied DC voltage, and the ordinate is the transverse deflection of the middle point of the beams. Figure 4 illustrates how the microbeam resonator loses stability. Before the DC voltage 


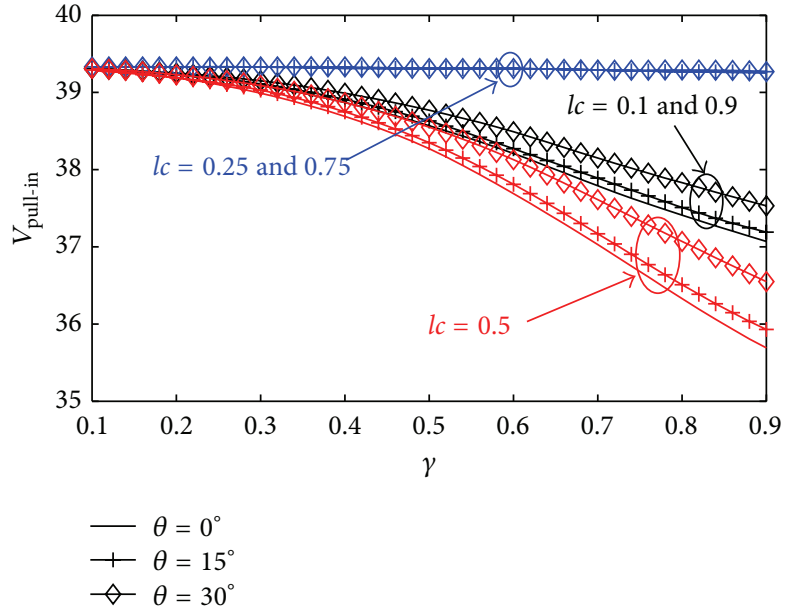

FIGURE 5: Variation of the pull-in voltage of the fixed-fixed microbeam with the crack depth ratio (for three crack positions and three slant angles).

increases to a certain value, it exhibits a steady increase trend in the deflection of center point of the beam. Once the DC voltage reaches a critical point, it enters the pull-in instability zone, where the deflection begins oscillating violently with the increasing of the DC voltage. This critical point indicates the pull-in voltage, and the corresponding displacement is the pull-in displacement.

By using the modified model proposed, the effects of the slant crack parameters on static pull-in voltage of the fixedfixed microbeam are investigated. The variation of the pull-in voltages of the cracked beam of different crack depth ratio (for three crack positions and three slant angles) is shown in Figure 5. It is shown that the crack position has a strong effect on the pull-in voltage. When the crack is near to the middle of the microbeam, especially for the higher crack depth ratios, the pull-in voltage is greatly reduced. And the position of $l c=0.25$ and 0.75 is more ineffective on the pull-in voltage than other positions. In addition, the effect of the slant angle on the pull-in voltage is appreciable. The pull-in voltage is increased slightly along with the increase of the angle. In addition, the detailed effect of the crack position on pull-in voltage of the beam is investigated and shown in Figure 6. As shown, interestingly, there are several extreme points located in $l c=0.25,0.5,0.75$ and two endpoints. As shown in the figure when the crack is located at $l c=0.25$ and 0.75 , it has the lowest effect on the pull-in voltage and when $l c=0.5$, the effect is greater, but endpoints are still lower. As shown, the rate of the variation is smaller in larger crack slant angle. The results gained above show that the crack position has more significant effect on the pull-in voltage of the beam than the crack depth ratio or the slant angel. And the slant angel exhibits a contrary effect on the pull-in voltage compared with the crack depth ratio.

4.3. Dynamic Response Analysis. The dynamic response of the microbeam described by (31) has been taken into account. The set of parameters applied in all the numerical simulations

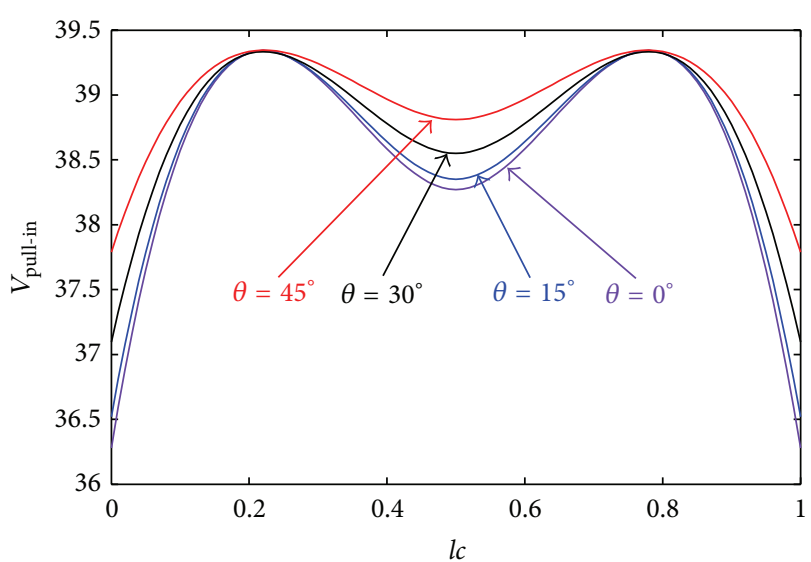

FIgURE 6: Comparison of the pull-in voltage of the slant-cracked microbeam with the crack position (for four slant angles).

TABLE 2: Parameters values for the solution and results values.

\begin{tabular}{|c|c|c|c|c|}
\hline Figure & \multicolumn{2}{|c|}{ Varying parameters } & $\begin{array}{l}\text { Resonant } \\
\text { frequency }\end{array}$ & Max-amplitude \\
\hline \multirow{3}{*}{ Figure 7} & \multicolumn{2}{|c|}{$l c=0.05$} & 21.63 & 0.4317 \\
\hline & \multicolumn{2}{|c|}{$l c=0.25$} & 22.34 & 0.4150 \\
\hline & \multicolumn{2}{|c|}{$l c=0.5$} & 22.06 & 0.4193 \\
\hline \multirow{3}{*}{ Figure 8(a) } & $\gamma=0.1$ & $l c=0.5$ & 22.34 & 0.4141 \\
\hline & $\gamma=0.5$ & $l c=0.5$ & 22.04 & 0.4188 \\
\hline & $\gamma=0.7$ & $l c=0.5$ & 21.43 & 0.4289 \\
\hline \multirow{3}{*}{ Figure 8(b) } & $\gamma=0.1$ & $l c=0.05$ & 22.33 & 0.4143 \\
\hline & $\gamma=0.5$ & $l c=0.05$ & 21.88 & 0.4235 \\
\hline & $\gamma=0.7$ & $l c=0.05$ & 21.09 & 0.4407 \\
\hline \multirow{2}{*}{ Figure 9(a) } & $\theta=0^{\circ}$ & $l c=0.5$ & 18.29 & 0.4399 \\
\hline & $\theta=23^{\circ}$ & $l c=0.5$ & 18.53 & 0.4377 \\
\hline \multirow{2}{*}{ Figure 9(a) } & $\theta=45^{\circ}$ & $l c=0.5$ & 20.88 & 0.4453 \\
\hline & $\theta=70^{\circ}$ & $l c=0.5$ & 21.56 & 0.4305 \\
\hline \multirow{4}{*}{ Figure 9(b) } & $\theta=0^{\circ}$ & $l c=0.05$ & 20.41 & 0.4561 \\
\hline & $\theta=23^{\circ}$ & $l c=0.05$ & 20.53 & 0.4532 \\
\hline & $\theta=45^{\circ}$ & $l c=0.05$ & 20.88 & 0.4453 \\
\hline & $\theta=70^{\circ}$ & $l c=0.05$ & 21.56 & 0.4305 \\
\hline \multirow{3}{*}{ Figure 10(a) } & \multicolumn{2}{|c|}{$V_{p}=1.0 \mathrm{~V}$} & 22.06 & 0.07172 \\
\hline & \multicolumn{2}{|c|}{$V_{p}=6.0 \mathrm{~V}$} & 22.04 & 0.2513 \\
\hline & \multicolumn{2}{|c|}{$V_{p}^{p}=6.0 \mathrm{~V}$} & 21.99 & 0.4317 \\
\hline \multirow{3}{*}{ Figure 10(a) } & \multicolumn{2}{|c|}{$v_{\mathrm{ac}}=0.01 \mathrm{~V}$} & 22.04 & 0.04188 \\
\hline & \multicolumn{2}{|c|}{$v_{\mathrm{ac}}=0.5 \mathrm{~V}$} & 22.04 & 0.2094 \\
\hline & \multicolumn{2}{|c|}{$v_{\mathrm{ac}}=0.1 \mathrm{~V}$} & 22.04 & 0.4188 \\
\hline
\end{tabular}

are $\theta=45^{\circ}, l c=0.5, \gamma=0.5, V_{p}=3.5 \mathrm{~V}$, and $v_{\mathrm{ac}}=0.1 \mathrm{~V}$. The resonance frequency and resonance amplitude are acquired by varying one of the parameters, as listed in Table 2 .

As plotted in Figures 7-10, the dynamic response curves of the first mode are acquired. The abscissa is the nondimensional resonance frequency, and the ordinate is the resonance amplitude. But the abscissa of the subgraphs in the graphs is the excitation frequency. As plotted, when the resonance 


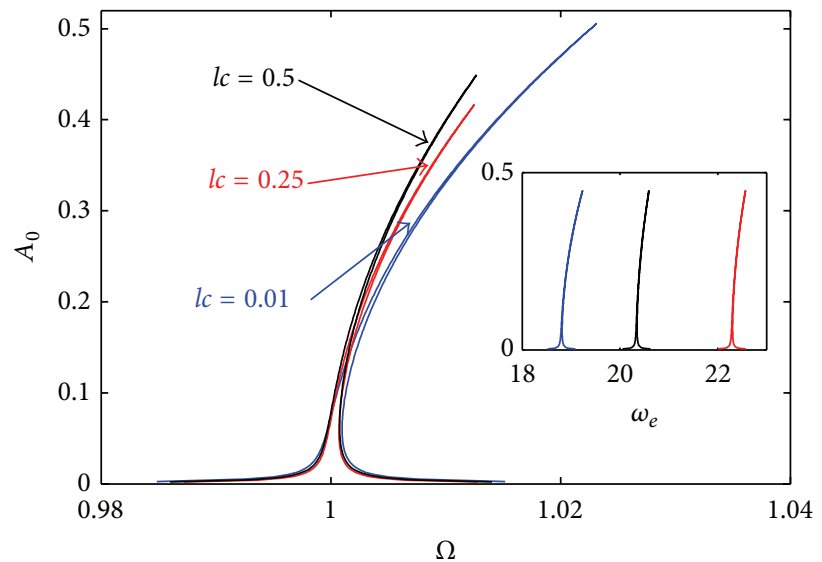

FIGURE 7: The effects of the crack position on the dynamic response of microbeams of different positions $l c$.

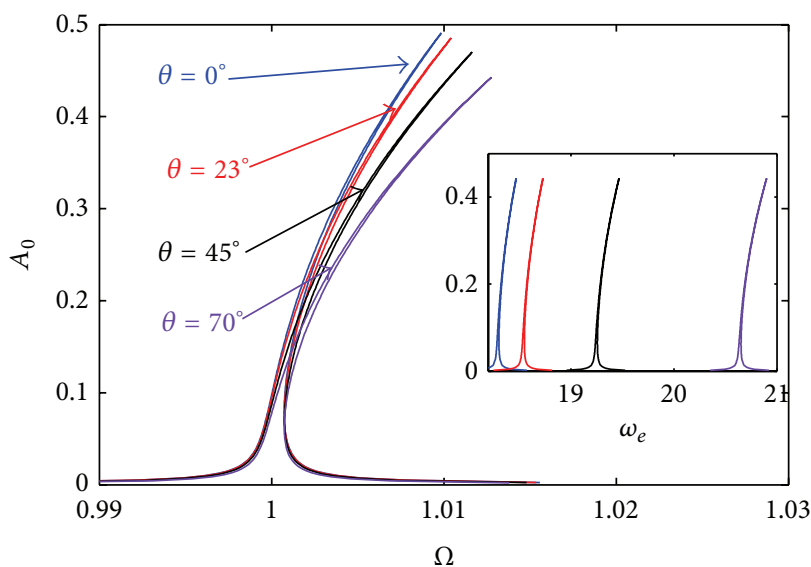

(a)

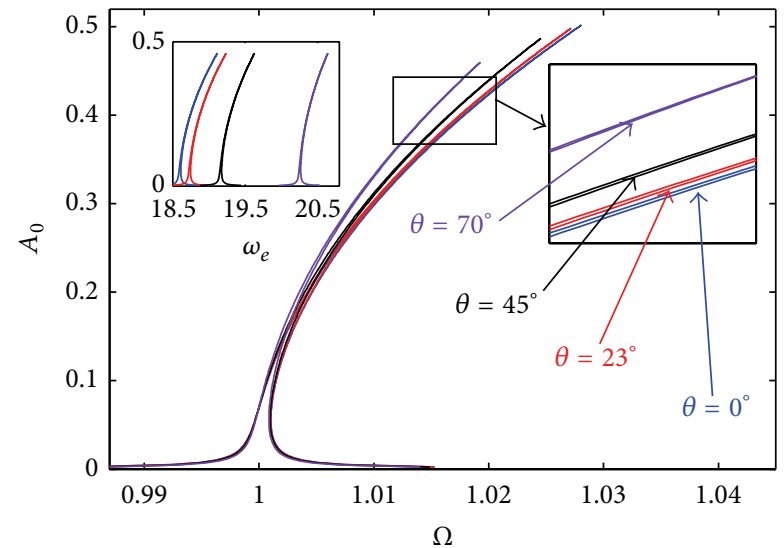

(b)

FIGURE 8: The effects of the slant angle of the crack on the dynamic response of microbeams. (a) $l c=0.5 ;$ (b) $l c=0.05$.

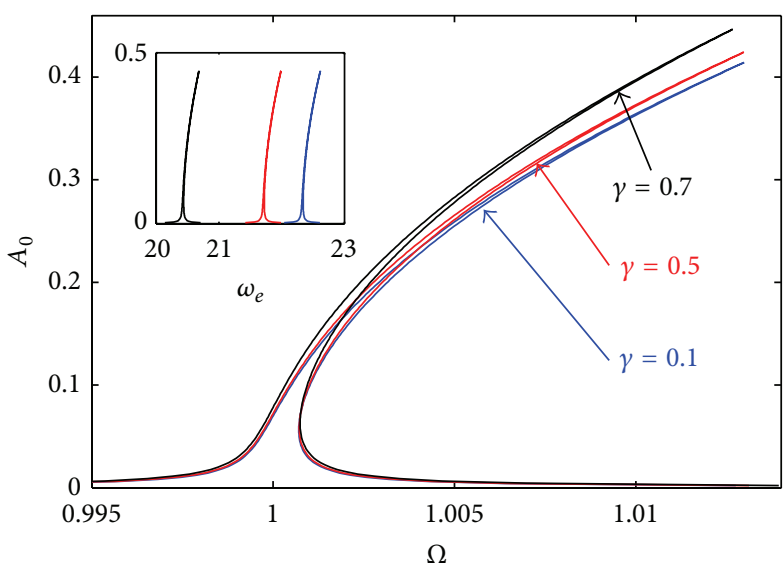

(a)

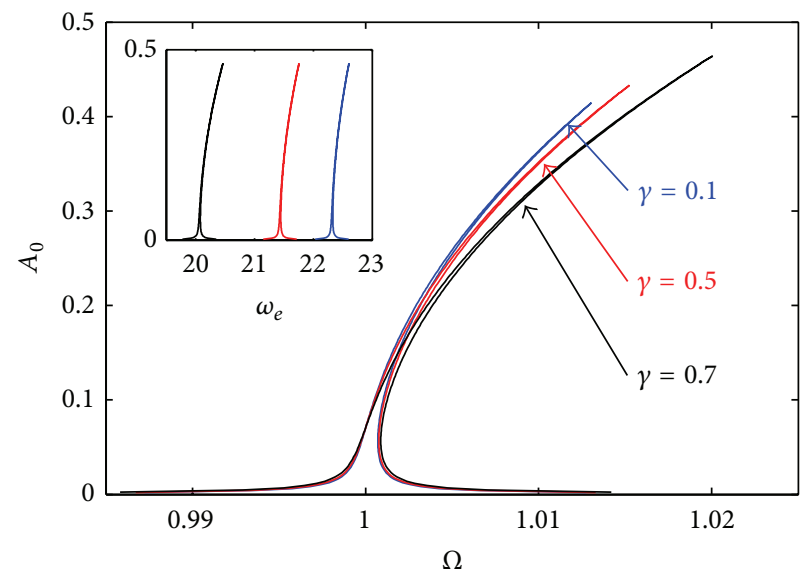

(b)

FIGURE 9: The effects of the crack depth ratio of the crack on the dynamic response of microbeams. (a) $l c=0.5$; (b) $l c=0.05$. 


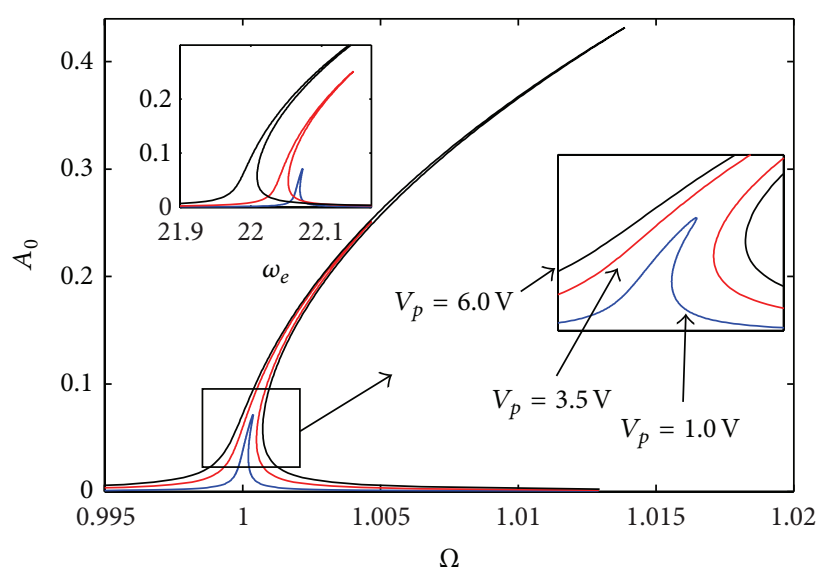

(a)

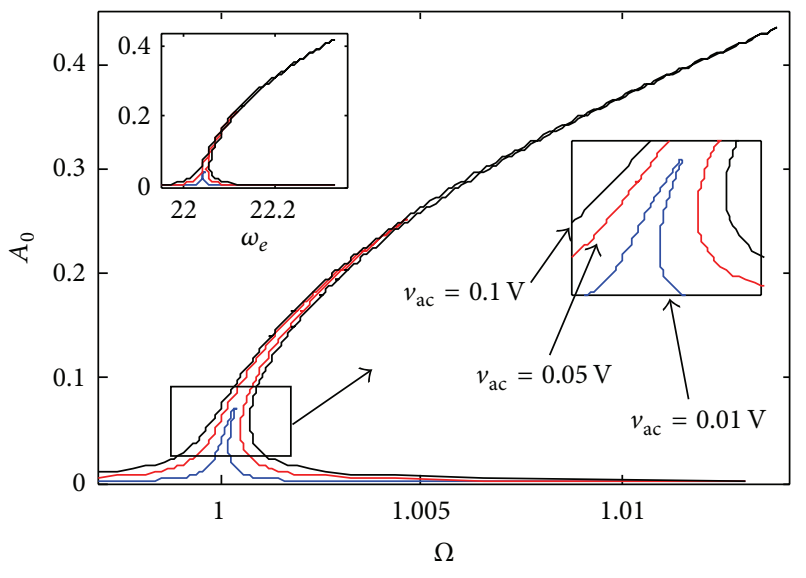

(b)

Figure 10: The effects of the external incentives on the dynamic response of microbeams. (a) DC voltage; (b) AC voltage.

frequency grows sufficiently large, the two branches of all the curves merge and produce a standard resonance peak with a hardening characteristic. A larger degree of curvature means the stronger hardening behavior, which points out that the nonlinearity strengthens.

Figure 7 illustrates the influence of the crack position on the frequency response of the microbeam. When the crack is located at the middle point of the beam, the nonlinear behavior of the system is not evident. As the crack approaches the fixed end, the influence of the nonlinearities becomes more obvious showing a typical hardening characteristic. At most frequencies, it is noted that the fact that the crack approaches the fixed end results in a decrease in the steadystate amplitude. For case of $l c=0.01$, the value of the nondimensional resonance frequency where the maximum amplitude occurs is bigger than the other two cases.

The influence of the slant angles of crack $\theta$ on the dynamic response differs at different crack locations as shown in Figure 8. When the crack is located at the center part of the microbeam $(l c=0.5$, Figure $8(a))$, the microbeam with a slant crack of a bigger angle displays a stronger nonlinearity but a less resonance amplitude at most frequencies. Increasing the slant angle decreases the maximum resonance amplitude and slightly increases the corresponding frequency. But if the crack is located at the fixed end of the microbeam $(l c=0.05$, Figure $8(b))$, the result becomes that the microbeam with a slant crack of a smaller angle depicts the stronger nonlinearity and the less resonance amplitude. Meanwhile, increasing the slant angle of the crack has no significant influence on the maximum resonance amplitude but decreases the corresponding frequency.

When it comes to the influence of the crack depth ratio $\gamma$ on the dynamic response, the characteristics displayed in Figure 9 are similar to what is seen in Figure 8. It is noted that a deeper crack affects the dynamic response of the microbeam in the same way with how a slant crack with a smaller slant angle does. Increasing the depth ratio of the crack located at the center part of the microbeam $(l c=0.5$, Figure 9(a)) weakens the nonlinear behavior of the microbeam and enlarges the resonance amplitude at most frequencies, as well as the maximum resonance amplitude, but it has no significant influence on the corresponding resonance frequency, contrary to the effects of the crack depth ratio on the response characteristics located at the fixed end $(l c=0.05$, Figure $9(\mathrm{~b}))$.

Figure 10 illustrates the influence of the external incentives on the dynamic response of microbeams including the DC voltage (Figure 10(a)) and the AC voltage (Figure 10(b)). As plotted in the two figures, it can be directly seen that increased external incentives result in an obvious increase in the steady-state amplitude at most frequencies as expected. As the value of the external incentives is decreased, the hardening behavior weakens. A resonance response will not be seen if the incentive values are significantly reduced. This result is in agreement with that found by Younis and Nayfeh [44].

\section{Conclusion}

In this work, the model of slant open crack is established, and it is applied into the continuous vibration equation of electrostatically actuated fixed-fixed microbeams. Analytic results of the natural frequency, the corresponding mode shapes, and the numerical results of the pull-in voltages are presented. By comparing the calculated results in different cases, the effects of the crack depth ratio, crack position, and the slant angle on the dynamic behaviors and the pullin voltages are investigated. It is shown that except for some special cases the first four nature frequencies decrease with the increase of the crack depth ratio, but the opposite change is displayed when the slant angle of the crack increases. In addition, the effect of the crack position on frequency differs in different modes. Furthermore, the influences of the crack position, slant angle, and the depth ratio on the pullin voltages are similar to those on frequencies, respectively. The pull-in voltage decreases the most when the crack is located at the middle point of the beams and the least when the crack is located at the quarter point. Finally, 
the dynamic response of the beam is investigated by employing the multiple scale perturbation theory. It is found that the response demonstrates a hardening characteristic, which is affected by the geometry parameters of the slant crack and the electric incentives. The nonlinearity of the resonance response gets enlarged when the crack position approaches the fixed end of the microbeam or the DC voltage and AC voltage value amplifies. The effects of the crack depth ratio and the slant angle on the dynamic response vary in different positions.

\section{Conflict of Interests}

The authors declare that there is no conflict of interests regarding the publication of this paper.

\section{Acknowledgments}

The authors gratefully acknowledge projects supported by the National Natural Science Foundation of China (no. 11322215), National Program for Support of Top-notch Young Professionals, the Fok Ying Tung Education Foundation (no. 141050), and the Innovation Program of Shanghai Municipal Education Commission (no. 15ZZ010).

\section{References}

[1] J. Tamayo, P. M. Kosaka, J. J. Ruz, Á. San Paulo, and M. Calleja, "Biosensors based on nanomechanical systems," Chemical Society Reviews, vol. 42, no. 3, pp. 1287-1311, 2013.

[2] L. M. Bellan, D. Wu, and R. S. Langer, "Current trends in nanobiosensor technology," Wiley Interdisciplinary Reviews: Nanomedicine and Nanobiotechnology, vol. 3, no. 3, pp. 229-246, 2011.

[3] S. Pourkamali and F. Ayazi, "Electrically coupled MEMS bandpass filters: part I: with coupling element," Sensors and Actuators A: Physical, vol. 122, no. 2, pp. 307-316, 2005.

[4] S. Pourkamali and F. Ayazi, "Electrically coupled MEMS bandpass filters: part II. Without coupling element," Sensors and Actuators A: Physical, vol. 122, no. 2, pp. 317-325, 2005.

[5] M. Fathalilou, A. Motallebi, H. Yagubizade, G. Rezazadeh, K. Shirazi, and Y. Alizadeh, "Mechanical behavior of an electrostatically-actuated microbeam under mechanical shock," Journal of Solid Mechanics, vol. 1, no. 1, pp. 45-57, 2009.

[6] A. Motallebi, M. Fathalilou, and G. Rezazadeh, "Effect of the open crack on the pull-in instability of an electrostatically actuated micro-beam," Acta Mechanica Solida Sinica, vol. 25, no. 6, pp. 627-637, 2012.

[7] H. C. Nathanson, W. E. Newell, R. A. Wickstrom, and J. R. Davis, "The resonant gate transistor," IEEE Transactions on Electron Devices, vol. 14, no. 3, pp. 117-133, 1967.

[8] D. Bernstein, P. Guidotti, and J. Pelesko, "Mathematical analysis of an electrostatically actuated MEMS device," in Proceedings of the Modelling \& Simulation of Microsystems, pp. 489-492, 2000.

[9] J. A. Pelesko, "Multiple solutions in electrostatic MEMS," in Proceedings of the International Conference on Modeling and Simulation of Microsystems (MSM '01), pp. 290-293, March 2001.

[10] W.-M. Zhang, H. Yan, Z.-K. Peng, and G. Meng, "Electrostatic pull-in instability in MEMS/NEMS: a review," Sensors and Actuators A: Physical, vol. 214, pp. 187-218, 2014.
[11] E. M. Abdel-Rahman, M. I. Younis, and A. H. Nayfeh, "Characterization of the mechanical behavior of an electrically actuated microbeam," Journal of Micromechanics and Microengineering, vol. 12, no. 6, pp. 759-766, 2002.

[12] V. Y. Taffoti Yolong and P. Woafo, "Dynamics of electrostatically actuated micro-electro-mechanical systems: single device and arrays of devices," International Journal of Bifurcation and Chaos, vol. 19, no. 3, pp. 1007-1022, 2009.

[13] F. Najar, S. Choura, S. El-Borgi, E. M. Abdel-Rahman, and A. H. Nayfeh, "Modeling and design of variable-geometry electrostatic microactuators," Journal of Micromechanics and Microengineering, vol. 15, no. 3, pp. 419-429, 2005.

[14] B.-G. Nam, S. Tsuchida, and K. Watanabe, "Fatigue crack growth driven by electric fields in piezoelectric ceramics and its governing fracture parameters," International Journal of Engineering Science, vol. 46, no. 5, pp. 397-410, 2008.

[15] T. G. Chondros, A. D. Dimarogonas, and J. Yao, "Vibration of a beam with a breathing crack," Journal of Sound and Vibration, vol. 239, no. 1, pp. 57-67, 2001.

[16] A. D. Dimarogonas, "Vibration of cracked structures: a state of the art review," Engineering Fracture Mechanics, vol. 55, no. 5, pp. 831-857, 1996.

[17] M. Rezaee and R. Hassannejad, "Free vibration analysis of simply supported beam with breathing crack using perturbation method," Acta Mechanica Solida Sinica, vol. 23, no. 5, pp. 459470, 2010.

[18] S. Caddemi, I. Caliò, and M. Marletta, “The non-linear dynamic response of the Euler-Bernoulli beam with an arbitrary number of switching cracks," International Journal of Non-Linear Mechanics, vol. 45, no. 7, pp. 714-726, 2010.

[19] A. Khorram, F. Bakhtiari-Nejad, and M. Rezaeian, "Comparison studies between two wavelet based crack detection methods of a beam subjected to a moving load," International Journal of Engineering Science, vol. 51, pp. 204-215, 2012.

[20] M. Donà, A. Palmeri, and M. Lombardo, "Exact closed-form solutions for the static analysis of multi-cracked gradientelastic beams in bending," International Journal of Solids and Structures, vol. 51, no. 15-16, pp. 2744-2753, 2014.

[21] M. Kisa and M. Arif Gurel, "Free vibration analysis of uniform and stepped cracked beams with circular cross sections," International Journal of Engineering Science, vol. 45, no. 2-8, pp. 364380, 2007.

[22] M. I. Friswell and J. E. T. Penny, "Crack modeling for structural health monitoring," Structural Health Monitoring, vol. 1, no. 2, pp. 139-148, 2002.

[23] T. G. Chondros, A. D. Dimarogonas, and J. Yao, "A continuous cracked beam vibration theory," Journal of Sound and Vibration, vol. 215, no. 1, pp. 17-34, 1998.

[24] S. Caddemi, I. Caliò, and F. Cannizzaro, "The influence of multiple cracks on tensile and compressive buckling of shear deformable beams," International Journal of Solids and Structures, vol. 50, no. 20-21, pp. 3166-3183, 2013.

[25] H. P. Lin, S. C. Chang, and J. D. Wu, "Beam vibrations with an arbitrary number of cracks," Journal of Sound and Vibration, vol. 258, no. 5, pp. 987-999, 2002.

[26] L. Rubio and J. Fernández-Sáez, "A note on the use of approximate solutions for the bending vibrations of simply supported cracked beams," Journal of Vibration and Acoustics, Transactions of the ASME, vol. 132, no. 2, 2010.

[27] M. Afshari and D. J. Inman, "Continuous crack modeling in piezoelectrically driven vibrations of an Euler-Bernoulli beam," Journal of Vibration and Control, vol. 19, no. 3, pp. 341-355, 2013. 
[28] M. H. Ghayesh, M. Amabili, and H. Farokhi, "Nonlinear forced vibrations of a microbeam based on the strain gradient elasticity theory," International Journal of Engineering Science, vol. 63, pp. 52-60, 2013.

[29] M. H. Ghayesh, H. Farokhi, and M. Amabili, "Nonlinear behaviour of electrically actuated MEMS resonators," International Journal of Engineering Science, vol. 71, pp. 137-155, 2013.

[30] S.-C. Sun, C.-W. Chung, C.-M. Hsu, and J.-H. Kuang, "The squeeze film damping effect on dynamic responses of microelectromechanical resonators," Applied Mechanics and Materials, vol. 284-287, pp. 1961-1965, 2013.

[31] H. O. Ekici and H. Boyaci, "Effects of non-ideal boundary conditions on vibrations of microbeams," Journal of Vibration and Control, vol. 13, no. 9-10, pp. 1369-1378, 2007.

[32] F. M. Alsaleem, M. I. Younis, and H. M. Ouakad, "On the nonlinear resonances and dynamic pull-in of electrostatically actuated resonators," Journal of Micromechanics and Microengineering, vol. 19, no. 4, Article ID 045013, 2009.

[33] Y. Lin and F. Chu, "The dynamic behavior of a rotor system with a slant crack on the shaft," Mechanical Systems and Signal Processing, vol. 24, no. 2, pp. 522-545, 2010.

[34] Q. Han, J. Zhao, and F. Chu, "Dynamic analysis of a geared rotor system considering a slant crack on the shaft," Journal of Sound and Vibration, vol. 331, no. 26, pp. 5803-5823, 2012.

[35] G. De Pasquale and A. Soma, "MEMS mechanical fatigue: effect of mean stress on gold microbeams," Journal of Microelectromechanical Systems, vol. 20, no. 4, pp. 1054-1063, 2011.

[36] A. K. Darpe, "Coupled vibrations of a rotor with slant crack," Journal of Sound and Vibration, vol. 305, no. 1-2, pp. 172-193, 2007.

[37] M. Kisa and J. Brandon, "Effects of closure of cracks on the dynamics of a cracked cantilever beam," Journal of Sound and Vibration, vol. 238, no. 1, pp. 1-18, 2000.

[38] A. K. Darpe, "Dynamics of a Jeffcott rotor with slant crack," Journal of Sound and Vibration, vol. 303, no. 1-2, pp. 1-28, 2007.

[39] J. P. Lynch, A. Partridge, K. H. Law, T. W. Kenny, A. S. Kiremidjian, and E. Carryer, "Design of piezoresistive MEMS-based accelerometer for integration with wireless sensing unit for structural monitoring," Journal of Aerospace Engineering, vol. 16, no. 3, pp. 108-114, 2003.

[40] M. I. Younis, E. M. Abdel-Rahman, and A. Nayfeh, "A reducedorder model for electrically actuated microbeam-based MEMS," Journal of Microelectromechanical Systems, vol. 12, no. 5, pp. 672-680, 2003.

[41] G. Rezazadeh, M. Fathalilou, R. Shabani, S. Tarverdilou, and S. Talebian, "Dynamic characteristics and forced response of an electrostatically-actuated microbeam subjected to fluid loading," Microsystem Technologies, vol. 15, no. 9, pp. 1355-1363, 2009.

[42] Z.-Y. Zhong, W.-M. Zhang, G. Meng, and J. Wu, "Inclination effects on the frequency tuning of comb-driven resonators," Journal of Microelectromechanical Systems, vol. 22, no. 4, pp. 865-875, 2013.

[43] B. S. M. Hasheminejad, B. Gheshlaghi, Y. Mirzaei, and S. Abbasion, "Free transverse vibrations of cracked nanobeams with surface effects," Thin Solid Films, vol. 519, no. 8, pp. 24772482, 2011.

[44] M. I. Younis and A. H. Nayfeh, "A study of the nonlinear response of a resonant microbeam to an electric actuation," Nonlinear Dynamics, vol. 31, no. 1, pp. 91-117, 2003. 


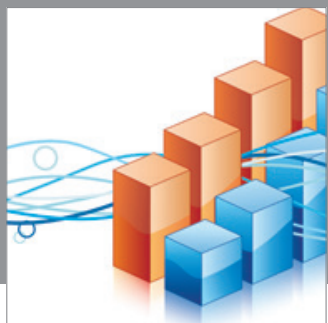

Advances in

Operations Research

mansans

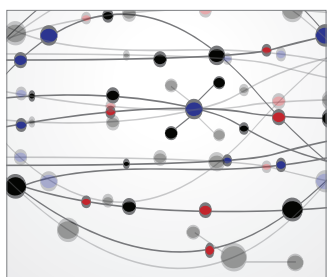

The Scientific World Journal
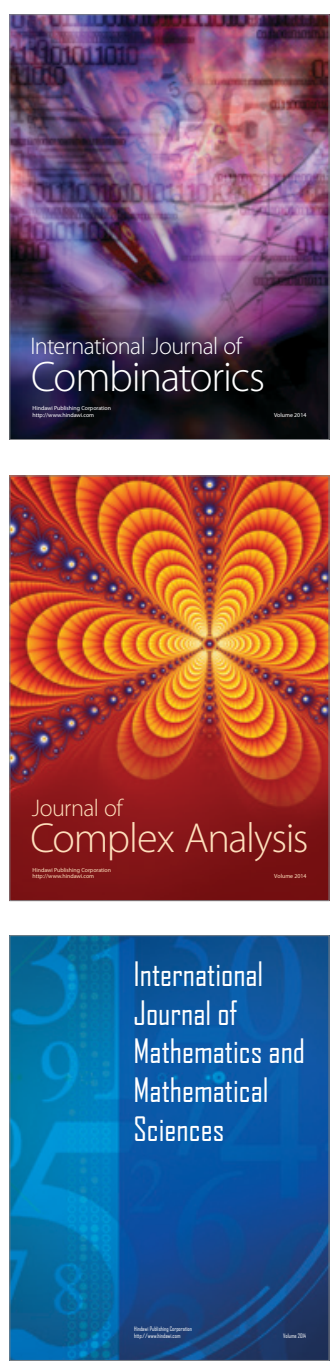
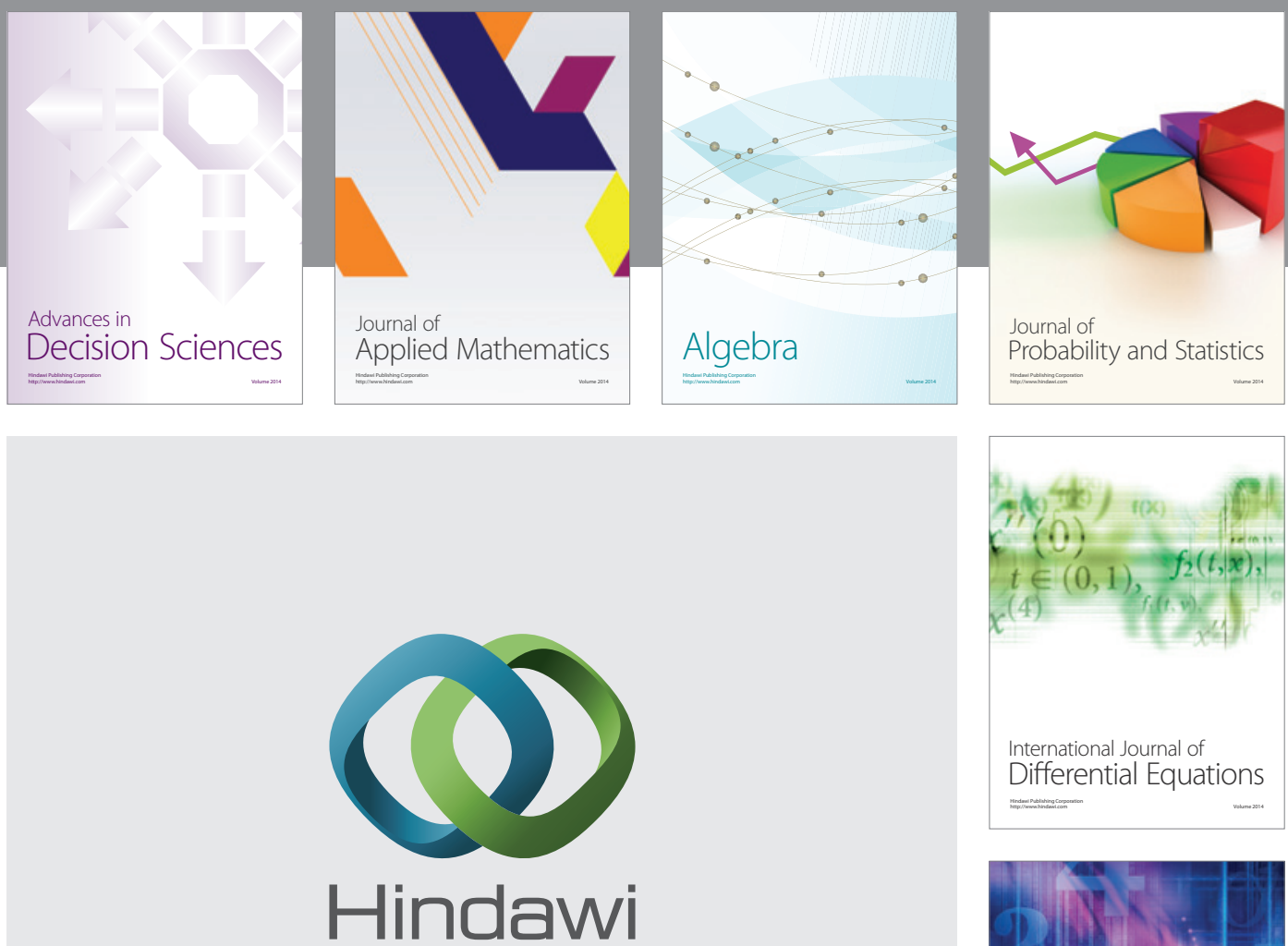

Submit your manuscripts at http://www.hindawi.com
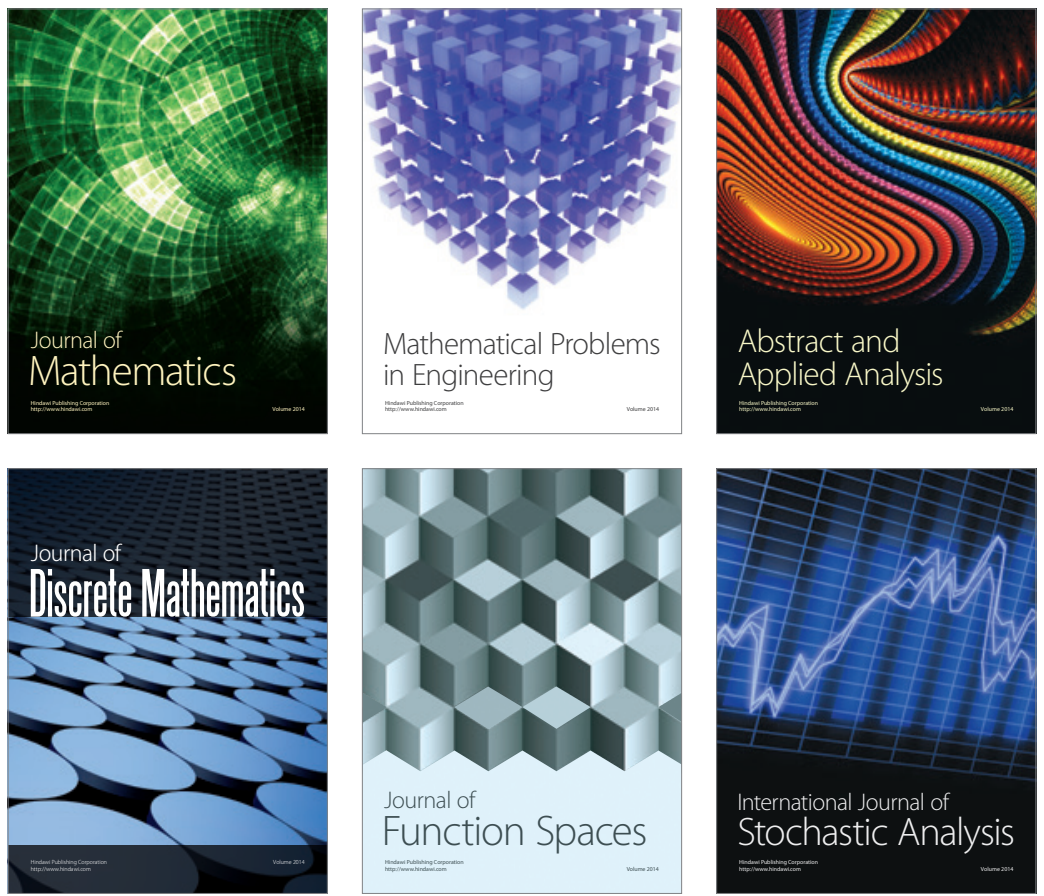

Journal of

Function Spaces

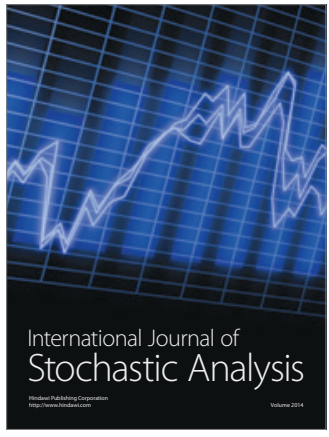

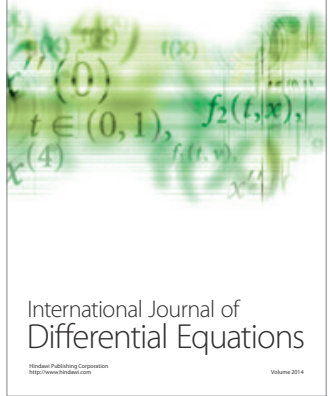
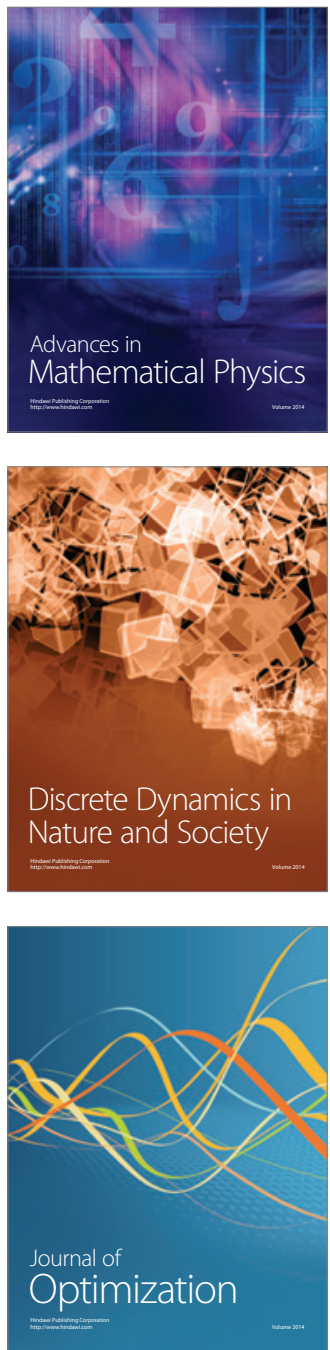